\title{
Determination of Hole Mobility in Polyethylene : First Principle Calculation Based on Marcus Theory
}

\author{
Masahiro Sato, Akiko Kumada, Kunihiko Hidaka \\ Department of Electrical Engineering \\ The University of Tokyo \\ 7-3-1, Hongo, Bunkyo-ku, Tokyo 113-8656, Japan
}

\author{
Toshiyuki Hirano, Fumitoshi Sato \\ Institute of Industrial Science \\ The University of Tokyo \\ 4-6-1, Komaba, Meguro-ku, Tokyo 153-8505, Japan
}

\begin{abstract}
Although degradation process of polymers is correlated with space charge, there remains a lack of understanding of charge transport phenomena and quantitative estimation of carrier mobility has not yet been accomplished. In this research, hole mobility in polyethylene $(\mathrm{PE})$ is calculated by means of standard density functional theory. It turns out that hole transfer in PE occurs in a "hopping regime". Moreover, estimated hole mobility is in reasonable agreement with experimental values. In addition, it is implied that the existence of carbonyl defect is likely to increase hole mobility which is consistent with experimental results at least for low density PE.
\end{abstract}

Keywords - first-principles calculation; density functional theory; charge transfer; hole; mobility; carbonyl; polyethylene; space charge; hopping

\section{INTRODUCTION}

Polyethylene (PE) is widely used as the insulation for transmission cables [1]. In order to improve the reliability and operating voltage, it is necessary to understand various high field effect in PE. Although degradation process of polymers is correlated with space charge which is formed in the material [2], there remains a lack of understanding of charge transport phenomena, despite extensive experimental efforts [3,4]. Recently first principle calculation has been utilized to clarify the nature of charge transfer in PE [5-13]. However, quantitative estimation of hole mobility, which is one of the most fundamental parameters required to understand charge transport in PE, has not yet been accomplished. Thus, it is necessary to strive towards the comprehensive understanding of charge transfer in organic insulators.

As is well known, evaluation of charge transfer between molecules is essential to understand conduction in organic materials $[14,15]$. It is suggested that band transport model is invalid for temperatures above $150 \mathrm{~K}$ even for charge transfer in semiconducting organic crystals such as aromatic hydrocarbons whose frontier orbitals are diffused to some extent $[16,17]$. This becomes much more notable when it comes to the evaluation of hole transfer in polyethylene whose Highest Occupied Molecular Orbital (HOMO) is localized within the molecule. In other words, charge transport in polymeric insulator can be regarded as a hopping of charge carriers between molecules under scattering by intramolecular vibration. Thus the purpose of this work is to evaluate hole transfer between molecules via hopping, thereby estimating hole mobility in PE.

\section{MARCUS THEORY}

Fig. 1 shows the schematic diagram of charge transfer between two molecules. Charge transfer between diabatic states is understood on the basis of Fermi's golden rule [18]. Assuming the Condon approximation, for high-temperature limit, the formula can be reduced to the well-known Marcus formula $[19,20]$;

$$
\begin{aligned}
k_{i f} & =\frac{2 \pi}{\hbar}\left|\left\langle\varphi_{i}|\hat{H}| \varphi_{f}\right\rangle\right|^{2} \frac{1}{\sqrt{4 \pi \lambda k_{B} T}} \exp \left(-\frac{\left(\Delta G^{0}+\lambda\right)^{2}}{4 \lambda k_{B} T}\right) \\
& \equiv \frac{2 \pi}{\hbar}\left(\mid \text { Transfer Integral }\left.\right|^{2}\right) \cdot(\text { FCWD }),
\end{aligned}
$$

where, $\varphi_{i}$ and $\varphi_{f}$ are diabatic initial and final states, respectively, $\Delta G^{0}$ is the free energy difference between the initial and final states, $\lambda$ is the reorganization energy; energy difference due to relaxation of geometry, and FCWD is called the Franck-Condon weighted factor.

\section{A. Transfer Integral}

Transfer integral is the most fundamental electronic factor in charge transfer process. There are several methods $[21,22]$ to compute this value. In this research, we have used the generalized Mulliken-Hush (GMH) method [23,24]. For twostate model, transfer integral is written as,
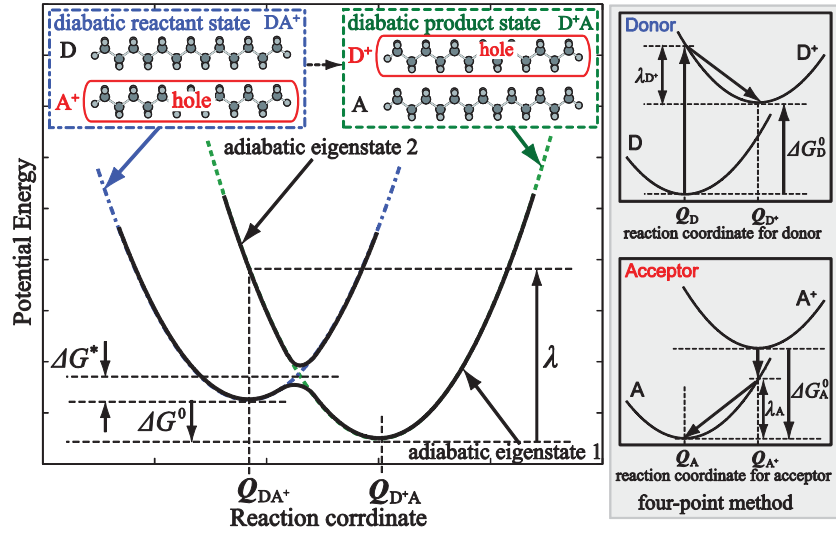

Fig. 1. Relation between adiabatic states and diabatic states for reactant and product. The diabatic state (broken line) represents the charge localized states. The adiabatic states (solid line) can be calculated by standard Density Functional Theory. The charge distribution of adiabatic states is not maintained along the reaction coordinate. The figure on the right shows the schematic diagram of four-point method. The value $\Delta G^{*} \equiv\left(\Delta G^{0}+\lambda\right)^{2} / 4 \lambda$ corresponds to the activation energy. Transfer integral, reorganization energy and free energy difference are called Marcus parameters. 


$$
\begin{aligned}
H_{i f} & =\left|\left\langle\varphi_{i}^{*}|\hat{H}| \varphi_{f}{ }^{*}\right\rangle\right| \\
& =\frac{\left(E_{2}-E_{1}\right)\left|\boldsymbol{\mu}_{12}\right|}{\sqrt{\left(\boldsymbol{\mu}_{1}-\boldsymbol{\mu}_{2}\right)^{2}+4 \boldsymbol{\mu}_{12}{ }^{2}}},
\end{aligned}
$$

where, $\varphi_{i}{ }^{*}$ and $\varphi_{f}{ }^{*}$ are estimated diabatic states before and after charge transfer, respectively, $\boldsymbol{\mu}_{1}-\boldsymbol{\mu}_{2}$ is adiabatic dipole moment difference, $\boldsymbol{\mu}_{12}$ is transition dipole moment, and $E_{2}-E_{1}$ is vertical excitation energy, i.e. estimated diabatic states are expressed by unitary transformation of adiabatic states $\psi$ as

$$
\begin{aligned}
\left(\begin{array}{c}
\varphi_{i}^{*} \\
\varphi_{f}^{*}
\end{array}\right) & =\left(\begin{array}{cc}
\cos \omega & -\sin \omega \\
\sin \omega & \cos \omega
\end{array}\right)^{-1}\left(\begin{array}{l}
\psi_{1} \\
\psi_{2}
\end{array}\right) \\
\tan \omega & =\frac{2\left|\boldsymbol{\mu}_{12}\right|}{\left|\boldsymbol{\mu}_{1}-\boldsymbol{\mu}_{2}\right|} .
\end{aligned}
$$

\section{B. Franck-Condon weighted density of states}

Reorganization energies and free energy differences were calculated by means four-point method $[25,26]$. As shown in Fig. 1, these values can be calculated directly from the adiabatic potential energy surface. The total reorganization energy and free energy difference is written as

$$
\begin{aligned}
\Delta G^{0} & =\Delta G_{D}{ }^{0}+\Delta G_{A}{ }^{0} \\
& =\left\{E_{D^{+}}\left(\boldsymbol{Q}_{D^{+}}\right)-E_{D}\left(\boldsymbol{Q}_{D}\right)\right\}+\left\{E_{A}\left(\boldsymbol{Q}_{A}\right)-E_{A^{+}}\left(\boldsymbol{Q}_{A^{+}}\right)\right\} \\
\lambda & =\lambda_{A}+\lambda_{D^{+}} \\
& =\left\{E_{A}\left(\boldsymbol{Q}_{A^{+}}\right)-E_{A}\left(\boldsymbol{Q}_{A}\right)\right\}+\left\{E_{D^{+}}\left(\boldsymbol{Q}_{D}\right)-E_{D^{+}}\left(\boldsymbol{Q}_{D^{+}}\right)\right\} .
\end{aligned}
$$

where, $E_{D}\left(\boldsymbol{Q}_{D}\right)$ and $E_{D+}\left(\boldsymbol{Q}_{D}\right)$ are the ground-state energy of neutral and cation state of " $\mathrm{D}$ " molecule at its optimized geometry at neutral state. Likewise, $E_{D}\left(\boldsymbol{Q}_{D^{+}}\right)$and $E_{D^{+}}\left(\boldsymbol{Q}_{D^{+}}\right)$are the energy of neutral and cation state at its optimal geometry at cation state.

\section{RESULTS AND DISCUSSION}

All the first-principles calculations were performed with Gaussian 09 package [27]. Other operations using the output of Gaussian, was done with MATLAB 2014b [28].

\section{A. Geometrical Structures}

We have chosen crystal structure (Fig. 2) and coreshell model (Fig. 3) for computing hole hopping rate. Coreshell model was developed by Huzayyin [8] to represent an approximation to amorphous PE with reasonable number of atoms. We have generated the coreshell model with and without carbonyl impurity. First geometry of a single PE oligomer was optimized at B3LYP/DZVP level with the combination with integral equation formalism polarizable continuum model (IEFPCM). The solvent for the model was heptane: oligomer of PE. Experimental lattice constants and angle $\phi$ were used to arrange the geometry-optimized PE chain. Since treating van der Waals (vdW) interactions with density functional theory (DFT) calculations still remains a challenge, we have computed the interaction energy curve at HartreeFock method, Second-order Møller-Plesset Perturbation Theory (MP2) and various DFT methods [29-30] in order to choose an appropriate exchange-correlation functional that can yield realistic structure. It is well known that, from a theoretical point of view, MP2 method can account for vdW interaction. Fig 4. shows the interaction energy computed with various theory level. Interaction energies calculated at functional with empirical dispersion takes its minimum around $d=4.7 \AA$, which is by far smaller than MP2/DZVP values $(d=5.2 \AA)$, while PBEPBE/DZVP (pure DFT method) values show good agreement with MP2/DZVP values. Since optimizing a coreshell model at MP2 level is almost impossible due to limitation of computational resources, coreshell model was optimized at PBEPBE/DZVP. In addition wB97XD/DZVP, which is a long-range corrected method was utilized for comparison purposes.

TABLE I shows the experimental and computed values of distance between centers of gravity of PE chains. Distances yielded by wB97XD are smaller than those calculated by PBEPBE. Moreover, wB97XD values are smaller than experimental values showing significant under estimation of $d$.

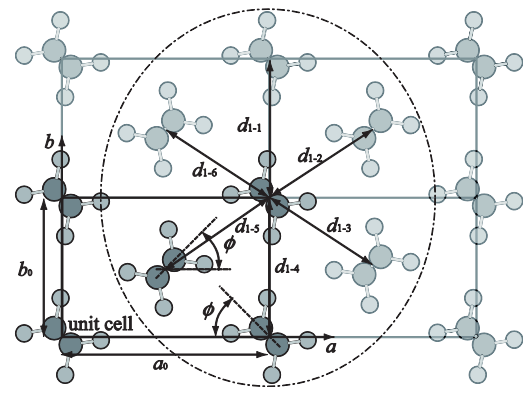

Fig. 2. Orthorhombic crystalline structure of PE along the axis of polymer chains. The solid frame corresponds to the unit cell in the $a-b$ plane. Seven PE chains in the broken circle is used as the initial coordination to generate coreshell model. The distance between the center of gravities of each PE chain $d$ is define as shown in this figure.

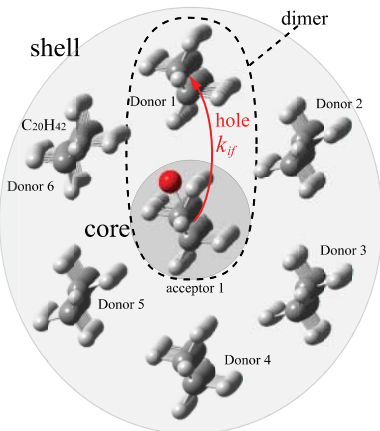

Fig. 3. Coreshell structure consisting of seven $\mathrm{C}_{20} \mathrm{H}_{42} \mathrm{PE}$ oligomers. As shown in this figure, the core chain and the surrounding chains are hereinafter termed "acceptor 1", "donor 1", "donor2", ..., "donor 6", respectively. A donor acceptor pair will be called dimer.

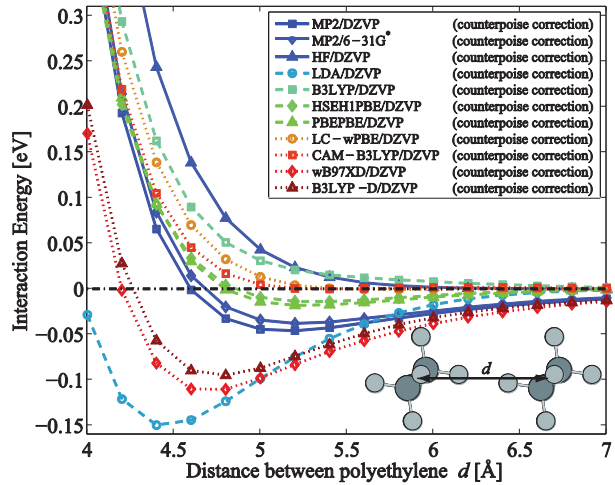

Fig. 4. Interaction Energy between two $\mathrm{C}_{6} \mathrm{H}_{14}$ molecules. Roll angle of $45^{\circ}$ is added from the face-to-face configuration; this relative coordination corresponds to dimer consisting of acceptor 1 and donor 1 . Basis set superposition error was corrected by counterpoise method. LCWPBE and CAM-B3LYP are long-range corrected functionals and wB97XD and B3LYP-D (D3 version of Grimme's dispersion with Becke-Johnson damping) are functionals with empirical dispersion. Similar tendancies were seen for interaction enegies with different dimer configuration or at the existence of carbonyl defect. 
Density of bulk PE estimated from coreshell model optimized at PBEPBE is around $0.90 \mathrm{~g} / \mathrm{cm}^{3}$, which is slightly smaller than that of low density PE (LDPE). Thus, we will assume that geometries obtained by PBEPBE is similar to LDPE with respect to intermolecular distance.

\section{B. Marcus parameters}

Transfer integral was calculated by (2). Regardless of the functionals used in geometry optimization, all calculations were performed at B3LYP/DZVP level of theory, in order to enable comparison of the computed values. Computed values of transfer integrals for crystal and coreshell structure are shown in TABLE II. When carbonyl impurity is added, increase in transfer integral in the direction of oxygen atom can be seen; transfer integral of dimer consisting of acceptor 1 and donor 6 with carbonyl impurity is roughly twice the value without carbonyls regardless of the theory level used to optimize the geometry. In order to account for this fact, diabatic molecular orbitals (MOs) before and after charge transfer is computed from (3) and visualized as shown in Fig. 5. It is clearly seen that diabatic MO without carbonyl impurity is localized within the molecule while that with carbonyl impurity is delocalized to some extent. This is due to the large contribution of $\pi$ orbital generated by $\mathrm{C}=\mathrm{O}$ bond to diabatic MO localized at acceptor 1. Enhancement of orbital overlap due to the diffusion of $\mathrm{MO}$ towards the neighboring molecule is one of the main reasons of the increase in transfer integral.

Computed values of reorganization energies and free energy differences of monomers are shown in TABLE III. Reorganization energy decreases as the number of carbon atoms of the PE chain increases. By extrapolating this trend, reorganization energy of monomers would converge to 100 $200 \mathrm{meV}$. Thus reorganization is by three orders of magnitude larger than transfer integral. This strongly indicates that hole transfer in PE occurs in a "hopping regime" not in a "band regime", since electronic coupling is dominant in the band regime while vibrational coupling, i.e. reorganization energy, is dominant in the hopping regime $[14,15]$.

In addition for small oligomer of $\mathrm{PE}$, reorganization energy decreases by adding carbonyl impurity; hole transfer is more likely to occur. Note that unlike most organic materials used in semiconductors, free energy difference of a dimer is generally nonzero. This is because hopping cite in polymeric materials are not identical; e.g. $\Delta G^{0}=\Delta G_{D}{ }^{0}+\Delta G_{A}{ }^{0}$ is around $300 \mathrm{mV}$ in the case of hole transfer from $\mathrm{C}_{20} \mathrm{H}_{42}$ to $\mathrm{C}_{10} \mathrm{H}_{22}$.

\section{Estimation of hopping rates and mobilities}

1) Crystal structure PE: Hopping rate for crystal structure is calculated as shown in TABLE IV. It should be noted that for crystal structure, free energy difference is zero; activation energy is $\lambda / 4$. When the hopping rate between dimer is given, diffusion constant can be evaluated as [16]

$$
D=\frac{1}{2 n} \sum_{j} \frac{d_{j}{ }^{2} k_{j}{ }^{2}}{\sum k_{l}},
$$

where, $n=2$ is the dimensionality, $k_{j}$ and $d$ are hopping rate and distance to the $j$ th neighbor, respectively. Thus the drift mobility can be evaluated through the Einstein relation: $\mu=e D / k_{B} T$, where $e$ is the elementary charge. Hole mobilities in $\mathrm{C}_{10} \mathrm{H}_{22}$ and $\mathrm{C}_{20} \mathrm{H}_{42}$ are calculated to be $6 \times 10^{-8}$ and $4 \times 10^{-6}$ $\mathrm{cm}^{2} /(\mathrm{Vs})$, respectively. In addition, roughly estimated hole
TABLE I. DISTANCE BETWEEN CENTERS OF GRAVITY OF MOLECULES

\begin{tabular}{|c|c|c|c|c|c|c|c|c|}
\hline \multirow{2}{*}{\multicolumn{2}{|c|}{ Geometry }} & \multirow{3}{*}{ 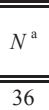 } & \multicolumn{6}{|c|}{ Distance between PE chains $[\AA]$} \\
\hline & & & \multirow{2}{*}{ 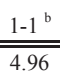 } & \multirow{2}{*}{ 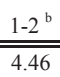 } & \multirow{2}{*}{ 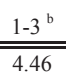 } & \multirow{2}{*}{$\frac{1-4^{b}}{4.96}$} & \multirow{2}{*}{$\frac{1-5^{b}}{4.46}$} & \multirow{2}{*}{ 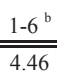 } \\
\hline $\mathrm{C}^{\text {Crystal }^{\mathrm{c}}}$ & Exp. $^{c}$ & & & & & & & \\
\hline Coreshell $^{\mathrm{b}}$ & PBEPBE & 10 & 5.34 & 4.87 & 4.86 & 5.34 & 4.87 & 4.87 \\
\hline Coreshell ${ }^{b}$ & wB97XD & 10 & 4.58 & 4.10 & 4.13 & 4.58 & 4.10 & 4.13 \\
\hline $\begin{array}{l}\text { Coreshell }{ }^{\mathrm{b}} \\
\text { (carbonyl) }\end{array}$ & PBEPBE & 10 & 5.14 & 4.77 & 4.97 & 5.50 & 4.87 & 4.80 \\
\hline $\begin{array}{l}\text { Coreshell }{ }^{\text {b }} \\
\text { (carbonyl) }\end{array}$ & wB97XD & 10 & 4.44 & 4.06 & 4.25 & 4.68 & 4.07 & 4.21 \\
\hline Coreshell $^{\mathrm{b}}$ & PBEPBE & 20 & 5.27 & 4.78 & 4.81 & 5.26 & 4.78 & 4.81 \\
\hline $\begin{array}{l}\text { Coreshell }{ }^{\mathrm{b}} \\
\text { (carbonyl) }\end{array}$ & PBEPBE & 20 & 5.18 & 4.79 & 4.98 & 5.31 & 4.83 & 4.88 \\
\hline
\end{tabular}

TABLE II. COMPUTED VALUES OF TRANSFER INTEGRAL

\begin{tabular}{|c|c|c|c|c|c|c|c|c|}
\hline \multirow{2}{*}{\multicolumn{2}{|c|}{ Geometry }} & \multirow{2}{*}{$N^{\mathrm{a}}$} & \multicolumn{6}{|c|}{ Transfer integral $H_{i f}[\mathrm{meV}]$} \\
\hline & & & $1-1^{\mathrm{b}}$ & $1-2^{b}$ & $1-3^{b}$ & $1-4^{\mathrm{b}}$ & $1-5^{b}$ & $1-6^{b}$ \\
\hline $\mathrm{Crystal}^{\mathrm{c}}$ & Exp. $^{c}$ & 10 & 1.4 & 3.6 & 3.6 & 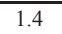 & 3.6 & 3.6 \\
\hline Crystal $^{\circ}$ & Exp. $^{\mathrm{c}}$ & 20 & 0.82 & 2.6 & 2.6 & 0.82 & 2.6 & 2.6 \\
\hline Coreshell ${ }^{\mathrm{b}}$ & PBEPBE & 10 & 0.63 & 0.78 & 1.2 & 0.63 & 0.78 & 1.1 \\
\hline Coreshell $^{\mathrm{b}}$ & wB97XD & 10 & 3.0 & 8.1 & 7.7 & 3.0 & 8.1 & 7.7 \\
\hline $\begin{array}{l}\text { Coreshell }{ }^{\mathrm{b}} \\
\text { (carbonyl) }\end{array}$ & PBEPBE & 10 & 0.45 & 0.43 & 1.0 & 0.44 & 0.57 & 2.1 \\
\hline $\begin{array}{l}\text { Coreshell1 } \\
\text { (carbonyl) }\end{array}$ & wB97XD & 10 & 1.2 & 4.7 & 5.4 & 1.8 & 2.7 & 15 \\
\hline Coreshell $^{\mathrm{b}}$ & PBEPBE & 20 & 0.3 & 0.92 & 0.87 & 0.3 & 0.92 & 0.87 \\
\hline $\begin{array}{l}\text { Coreshell }{ }^{\mathrm{b}} \\
\text { (carbonyl) }\end{array}$ & PBEPBE & 20 & 0.65 & 0.27 & 0.36 & 0.17 & 0.35 & 1.4 \\
\hline
\end{tabular}

TABLE III. COMPUTED VALUES OF FREE ENERGY DIFFERENCES AND REORGANIZATION ENERGIES FOR MONOMERS

\begin{tabular}{cccccc}
\hline \hline \multirow{2}{*}{ Impurity } & \multirow{2}{*}{$N^{\mathrm{a}}$} & \multicolumn{2}{c}{ Free energy difference [eV] } & \multicolumn{2}{c}{ Reorganization energy [eV] } \\
& & $\Delta G_{D}{ }^{0}$ & $\Delta G_{A}{ }^{0}$ & $\lambda_{D}$ & $\lambda_{A}$ \\
\hline \hline none & 4 & 9.09 & -9.09 & 1.01 & 1.37 \\
none & 10 & 8.35 & -8.35 & 0.540 & 0.603 \\
none & 20 & 8.03 & -8.03 & 0.311 & 0.348 \\
carbonyl & 10 & 7.97 & -7.97 & 0.177 & 0.215 \\
carbonyl & 20 & 7.79 & -7.79 & 0.158 & 0.182 \\
\hline \hline
\end{tabular}

TABLE IV. HOPPING RATE FOR CRYSTAL STRUCTURE (AT 300K)

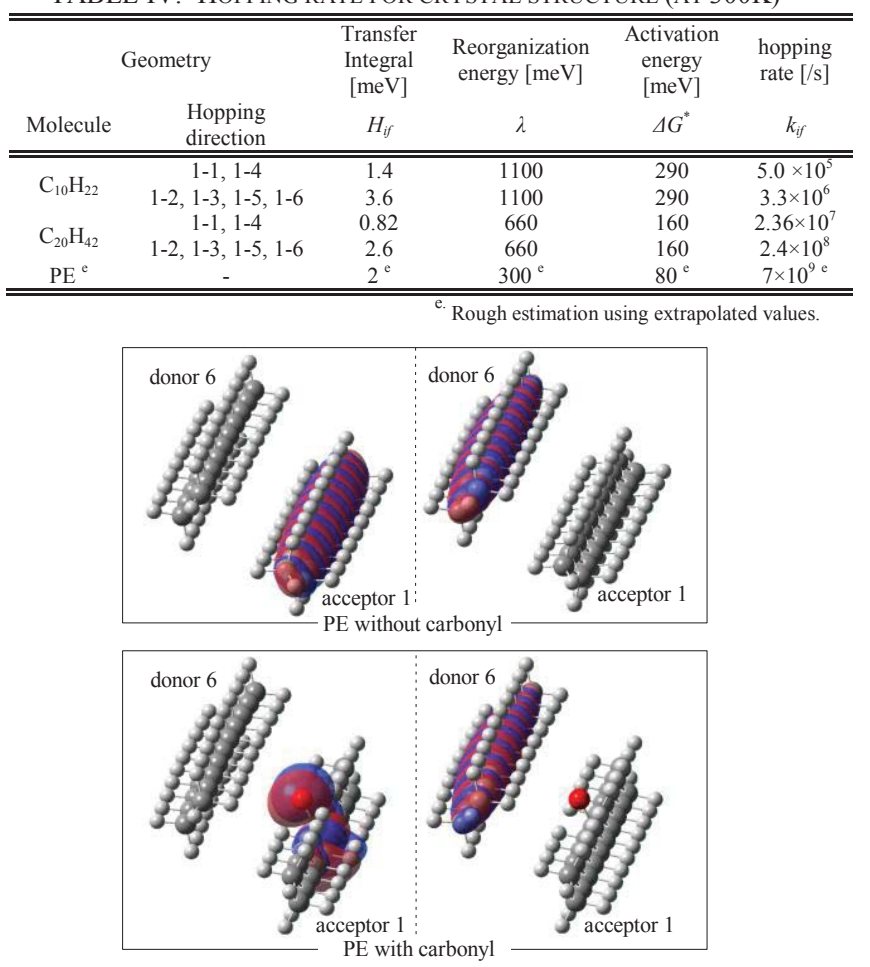

Fig. 5. Diabatic MOs $\left(\varphi_{i} / \varphi_{f}\right.$ : left/right $)$. It can be seen that estimated diabatic states are similar to HOMO of each monomer. (Isosurface values: 0.02 ) 
mobility obtained by extrapolating transfer integrals and reorganization energy with respect to number of carbon atoms per chain yields the value around $10^{-3}$.

2) Amorphous region and effect of carbonyl impurities in $P E$ : Hopping rate for amorphous region and the effect of carbonyl impurities are discussed qualitatively. Relation between activation energy, and reorganization energies and free energy differences is shown in Fig. 6. As shown in Fig. 6 activation energy is not symmetric with respect to inversion of free energy differences. This means that $k_{\mathrm{DA}}$ is not equal to $k_{\mathrm{AD}}$ and holes tends to remain at long PE chains rather than short ones, and if the length of PE chain is identical, holes prefer to remain at PE chains with carbonyl impurities. These results are in line with the conclusion of former studies [8-10]. If hopping rate from $\mathrm{D}$ to $\mathrm{A}$ is larger than that of $\mathrm{A}$ to $\mathrm{D}$, it is natural to assume that effective hopping rate is dominated by the smaller one. This may not hold true if there are several hopping paths for a single hopping cite. However, under this assumption, the effective activation energies are those corresponding to positive values of free energies, which is expected to be around 100-300 meV (Fig. 6). Strict computation of macroscopic activation energy is beyond the scope of this work. However, the roughly estimated values are in reasonable agreement with experimental values; activation energy of hole mobility in LDPE, measured by time-of-flight method is $320 \mathrm{meV}$ [32].

With regard to transfer integrals, considering the fact that density of coreshell geometry obtained by PBEPBE is similar to amorphous region, and transfer integral is mainly determined by the distance between PE chains, we may assume that transfer integral obtained at PBEPBE geometry represents that of amorphous region. Since transfer integrals obtained at PBEPBE geometry is roughly one-third of that at crystal structure, according to (1), hopping rate of amorphous region is one order of magnitude smaller than that of crystal structure. Regarding the effect of carbonyl impurity, hopping rate between acceptor 1 and donor 6 is increased by a factor of 4 whereas hopping rate between acceptor 1 and other donors are slightly decreased. If we take (5) as a first approximation, hole mobility will increase due to carbonyl impurity.

These results indicate that hole hopping rate in amorphous region is lower compared to that in crystal, both due to smaller transfer integrals and due to larger activation energies.

\section{CONCLUSION}

Hole mobility in PE was evaluated quantitatively by means of first-principles calculation. Computed Marcus parameters strongly indicated that hole transfer in PE occurs in a "hopping regime" not in a "band regime". It was indicated that hole hopping rate in amorphous region is lower compared to that in crystal, both due to smaller transfer integrals and due to larger activation energies. In addition, it was shown that carbonyl impurity has a potential to increase hole mobility; one of the factors is the increase in transfer integral. The computed value of activation energy of hole mobility was in good agreement with experimental results.

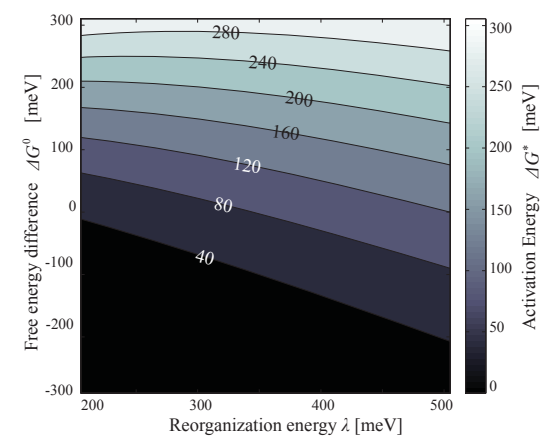

Fig. 6. Relation between activation energy, and reorganization energies and free energy differences. Each values is varied between 200 to 500 and -300 to $300 \mathrm{meV}$ respectively.

\section{REFERENCES}

[1] M. P. Bahrman and B. K. Johnson, "The ABCs of HVDC transmission technologies", IEEE PE Mag., 5 2, pp.32-44 (2007)

[2] L. A. Dissado et al., "The Incorporation of Space Charge Degradation in the Life Model for Electrical Insulating Materials", IEEE TDEI, 2 6, 1147 (1995)

[3] Y. Li, T. Takada, and N. Takasu," Experimental observation of charge transport and injection in XLPE at polarity reversal", J. Phys. D 26, 986 (1992)

[4] M. Ieda, IEEE TDEI, "Electrical conduction and carrier traps in polymeric materials", EI-19 3, pp.162-178 (1984)

[5] M. Meunier and N. Quirke, "Molecular modeling of electron trapping in polymer insulators", J. Chem. Phys., 113369 (2000)

[6] M. Meunier et al., "Characterisation of charge carrier traps in polymeric insulators", IEEE CEIDP, pp.21-24 (2000)

[7] M. Meunier et al., "Molecular modeling of electron traps in polymer insulators: Chemical defects and impurities", J. Chem. Phys., 1152876 (2001)

[8] A. Huzayyin et al., "Density Functional Analysis of Chemical Impurities in Dielectric Polyethylene", IEEE TDEI, 17 3, pp.920-925 (2010)

[9] A. Huzayyin et al., "Quantum Mechanical Studies of Carbonyl Impurities in Dielectric Polyethylene", IEEE TDEI, 17 3, pp.926-930 (2010)

[10] A. Huzayyin et al., "Density Functional Theory Analysis of the Effect of Iodine in Polyethylene", IEEE TDEI, 18 2, pp.471-477 (2011)

[11] M. Unge et al., "Electronic structure of polyethylene-Crystalline and amorphous phases of pure polyethylene and their interfaces", IEEE CEIDP, pp.525-530 (2012)

[12] M. Unge et al., "Space charges and deep traps in polyethylene-Ab initio simulations of chemical impurities and defects", IEEE ICSD, pp.935-939 (2013)

[13] Y. Sun et al., "Monte Carlo Studies of Hot Electron Transport and High Field Degradation", IEEE CEIDP, pp.15-18 (2014)

[14] T. Holstein, "Studies of polaron motion : Part I. The molecular-crystal model", Ann. Phys. 8, pp.325-342 (1959)

[15] T. Holstein, "Studies of polaron motion : Part II. The "small" polaron", Ann. Phys. 8, pp.343-389 (1959)

[16] W. Deng and W. A. Goddard III, "Predictions of Hole Mobilities in Oligoacene Organic semiconductors from quantum mechanical calculations", J. Phys. Chem. B, 1088614 (2004)

[17] L. Wang et al., "Multiscale Study of Charge Mobility of Organic Semiconductor with Dynamic Disorders", Phys. Chem. Chem. Phys., 123309 (2010)

[18] P. A. M. Dirac, "The Quantum Theory of Emission and Absorption of Radiation", Proc. Roy. Soc. Lond. A, 114, 767, pp.243-265 (1927)

[19] R. A. Marcus, "Electron transfer reactions in chemistry. Theory and experiment", Rev. Mod. Phys. 65, 599 (1993)

[20] E. F. Vallev et al., "Effect of Electronic Polarization on Charge-Transport Parameters in Molecular Organic Semiconductors", JACS, 128 pp.9882-9886 (2006)

[21] T. Fujita et al., "Ab Initio Molecular Orbital Model of Scanning Tunneling Microscope", J. Chem. Phys., 1042410 (1996)

[22] A. Troisi and G. Orlandi, "Hole Migration in DNA: a Theoretical Analysis of the Role of Structural Fluctuations", J. Phys. Chem. B, 1062093 (2002)

[23] R. J. Cave and M. D. Newton, "Calculation of Electronic Coupling Matrix Elements for Ground and Excited State Electron Transfer Reactions: Comparison of the Generalized Mulliken-Hush and Block Diagonalization Methods", J. Chem. Phys., 106, pp. 9213-9226 (1997)

[24] R. J. Cave et al., "Reduced Electronic Spaces for Modeling Donor/Acceptor Interactions", J. Phys. Chem. B, 11414631 (2010)

[25] S. F. Nelsen et al.," Estimation of inner shell Marcus terms for amino nitrogen compounds by molecular orbital calculations", JACS, 109 pp.678-682 (1987)

[26] Q. Wu and T. V. Voorhis, "Direct Calculation of Electron Transfer Parameters through Constrained Density Functional Theory", J. Phys. Chem. A, 1109212 (2006)

[27] Gaussian 09, Revision D.01, M. J. Frisch et al., Gaussian, Inc., Wallingford CT, 2010 .

[28] MATLAB 2014b Natick, Massachusetts: The MathWorks Inc., 2014

[29] O. A. Vydrov and G. E. Scuseria, "Assessment of a long range corrected hybrid functional", J. Chem. Phys., 125234109 (2006)

[30] A S. Grimme et al., "A consistent and accurate ab initio parameterization of density functional dispersion correction (DFT-D) for the 94 elements H-Pu", J. Chem. Phys., 132154104 (2010)

[31] P. W. Teare, "The crystal structure of orthorhombic hexatriacontane, $\mathrm{C}_{36} \mathrm{H}_{74}$ ", Acta Cryst. 12294 (1959)

[32] K. Yoshino et al.,"Carrier mobilities and breakdown in halogen doped polymers", J. Appl. Phys., 512714 (1980) 